\title{
Option pricing with transaction costs using a Markov chain approximation
}

\author{
Michael Monoyios* \\ Department of Economics and Finance, Brunel University, Uxbridge, Middx UB8 3PH, UK
}

\begin{abstract}
An efficient algorithm is developed to price European options in the presence of proportional transaction costs, using the optimal portfolio framework of Davis (in: Dempster, M.A.H., Pliska, S.R. (Eds.), Mathematics of Derivative Securities. Cambridge University Press, Cambridge, UK). A fair option price is determined by requiring that an infinitesimal diversion of funds into the purchase or sale of options has a neutral effect on achievable utility. This results in a general option pricing formula, in which option prices are computed from the solution of the investor's basic portfolio selection problem, without the need to solve a more complex optimisation problem involving the insertion of the option payoff into the terminal value function. Option prices are computed numerically using a Markov chain approximation to the continuous time singular stochastic optimal control problem, for the case of exponential utility. Comparisons with approximately replicating strategies are made. The method results in a uniquely specified option price for every initial holding of stock, and the price lies within bounds which are tight even as transaction costs become large. A general definition of an option hedging strategy for a utility maximising investor is developed. This involves calculating the perturbation to the optimal portfolio strategy when an option trade is executed.
\end{abstract}

(C) 2003 Elsevier B.V. All rights reserved.

JEL classification: C61; G11; G13

Keywords: Option pricing; Transaction costs; Utility maximisation; Singular stochastic control; Markov chain approximation

\section{Introduction}

The celebrated Black and Scholes (1973) option pricing methodology is not applicable in the presence of transaction costs on trading the underlying stock. This is

\footnotetext{
* Tel.: +44-1895-203181; fax: +44-1895-203384.

E-mail address: michael.monoyios@brunel.ac.uk (M. Monoyios).
} 
because it relies on the implementation of a continuously rebalanced portfolio strategy (to replicate the option payoff) that would be excessively costly in the face of any market frictions. Since perfect replication is not possible it is necessary to look for alternative criteria to determine fair valued derivative prices and, if possible, hedging strategies. There is an unavoidable trade-off between the transaction costs incurred in portfolio rebalancing and the tightness of the valuation bounds. Reducing the hedging error generally incurs large transaction costs and results in unrealistically wide valuation bounds. The question arises as to what is the optimal valuation and hedging policy, consistent with an investor's risk management objectives.

A number of methods have been suggested to solve this problem. The natural alternative to continuous hedging policies for options is to introduce a discrete timescale in which transactions take place. This is the course taken by Leland (1985) and Boyle and Vorst (1992). Both these models result in Black-Scholes type formulae for bounds on the value of the option, with an adjusted volatility which depends directly on the exogenously specified revision frequency. The hedging error is reduced if portfolio rebalancing occurs more frequently, but the pricing bounds become much wider. Moreover, restricting the form of the hedging strategy to portfolio rebalancing at fixed time intervals may not always be the optimum method of managing the risk from an option trade.

Bensaid et al. (1992), Edirisinghe et al. (1993) and Boyle and Tan (1994) replaced the replication strategy with a "super-replicating strategy" in which the hedging portfolio is only required to dominate, rather than replicate, the option payoff at maturity. In a discrete-time setting it can sometimes be cheaper to dominate a contingent claim than to replicate it. However, as conjectured by Davis and Clark (1994) and subsequently proven by Soner et al. (1995) and Cvitanić et al. (1999), in the continuous-time limit the cheapest super-replicating strategy for a European call option is the trivial strategy of buying one share of the underlying stock and holding it to maturity. This illustrates a fundamental feature of option hedging under transaction costs, namely that eliminating all risk results in unrealistically wide valuation bounds.

Hodges and Neuberger (1989) recognised that a valuation method which incorporates some element of optimality, in the form of the agent's utility maximisation objective, is perhaps the most promising path to follow in designing effective option hedging policies. This point was also made by Dumas and Luciano (1991). By comparing the utility achieved with and without the obligations of an option contract, Hodges and Neuberger specified reservation bid and asking prices for an option by requiring that the same utility is achieved whether an option trade has been entered into or not. This approach was further developed by Davis et al. (1993), Clewlow and Hodges (1997), Constantinides and Zariphopoulou (1999) and Musiela and Zariphopoulou (2001). Alternative criteria for determining an option hedging policy include quadratic criteria such as local risk minimisation, studied by Mercurio and Vorst (1997) and Lamberton et al. (1998), and minimisation of "shortfall risk", developed by Föllmer and Leukert (2000).

Davis (1997) suggested a utility maximisation approach to valuing options in a general situation in which replication is either impossible or unfavourable. Davis derived a general option pricing formula for a "fair" option value, determined by balancing 
the marginal utility from diverting an infinitesimal fraction of the initial wealth into the options market with that from refraining from trading options and instead investing optimally in the underlying asset market.

In this paper we numerically study the optimal pricing procedure suggested by Davis (1997). We develop an associated definition of a hedging strategy implied by the new method, and compute option prices and hedging strategies under proportional transaction costs. Davis' formula, which is reminiscent of a classical representative agent asset pricing formula, has not (until now) been tested numerically to see what implications it has for option values and hedging strategies in the presence of market frictions. Karatzas and Kou (1996) have applied this approach in frictionless markets to the situation in which there are constraints on the investor's portfolio choices, whereas we consider markets that are otherwise complete, but option replication is rendered unfavourable by trading costs.

Our numerical results indicate that the new methodology inherits realistic and appealing features from the solution of the investor's basic portfolio selection problem. The presence of frictions in a financial market qualitatively changes the nature of the optimal trading strategy, in that it specifies a region of the portfolio state space in which it is optimal not to transact, whilst outside this region the optimal action is to either buy or sell the risky asset. When the investor's portfolio holdings lie outside the no-transaction region, the derived option value is either the optimal bid or ask price. Alternatively, if the agent's stock-bond holdings lie within the no-transaction region, then the option value lies strictly within the bid-ask spread, and is uniquely specified given the initial portfolio holdings. Trading an option at any price within the optimal bid-ask interval results in a negligible effect on the investor's utility. However, trading at a price outside the bid-ask interval drastically reduces (or increases, if the investor is on the favourable side of the trade) the achievable utility compared with the scenario in which an option is not traded.

We also make comparisons with the replication approach of Leland (1985). In general, we find that the optimal valuation method produces much tighter bounds on option prices than can be obtained by any strategy which attempts to eliminate risk by replication, and this effect is most marked for large transaction costs. In essence, the option trader is able to incorporate some risk in his options portfolio, which is unavoidable in the face of market frictions.

The pricing methodology has the desirable feature that one only has to solve the investor's basic portfolio selection problem to price options. This is in contrast to previous attempts to embed the pricing problem in a utility maximisation framework, which require the solution of a much more difficult optimisation problem involving the credit or debit of an option payoff to the investor's portfolio at the option maturity date. We develop an efficient algorithm to price options in our framework using a Markov chain approximation technique of the type pioneered by Kushner (1990).

The paper is organised as follows. In Section 2 we set up a portfolio selection scenario, in which the optimal strategy to maximise expected utility of wealth at a finite horizon time is sought. We also formulate the alternative optimisation problem which results if a small amount of the initial wealth is diverted into the purchase or sale of European options. We illustrate the various ways that such stochastic optimal 
control problems can be used to specify an option price, and we state Davis' (1997) general option pricing formula. Then we give a general definition of an option hedging strategy for a utility maximising investor who has the choice of diverting some initial wealth into the options market. In Section 3 we consider a specific market model with transaction costs. We illustrate the properties of the solution to the portfolio choice problem, and of Davis' pricing formula. In Section 4 we specialise to the case of an exponential utility function, and develop an efficient numerical option pricing algorithm. In Section 5 we present numerical solutions for option prices and hedging strategies. Section 6 concludes and suggests directions for further research. An Appendix contains a derivation of some results used in the implementation of our numerical algorithm.

\section{Portfolio selection and option valuation}

We shall utilise a finite time interval $[0, T]$, where $T$ will correspond to the maturity of a European option. Consider an investor with concave utility function $U$, starting at time $t \in[0, T]$ with cash endowment $x$, and holding $y$ shares of a stock whose price is $S$. The investor trades a dynamic portfolio whose value at time $u>t$ is $W_{t, S, x, y}^{\pi}(u)$ when he or she uses the trading strategy $\pi$ and starts in the state $(t, S, x, y)$. The wealth $W_{t, S, x, y}^{\pi}(u)$ consists of $X_{t, S, x, y}^{\pi}(u)$ dollars in cash and $Y_{t, S, x, y}^{\pi}(u)$ shares of stock, whose price at time $u$ is $S(u)$, so that

$$
W_{t, S, x, y}^{\pi}(u)=X_{t, S, x, y}^{\pi}(u)+Y_{t, S, x, y}^{\pi}(u) S(u) .
$$

The investor's objective is to maximise expected utility of wealth at time $T$. We assume that the investor does not consume any wealth, which is a reasonable assumption for a trader in a financial institution. Denote the investor's maximum utility by

$$
V(t, S, x, y)=\sup _{\pi} \mathbb{E}_{t}\left[U\left(W_{t, S, x, y}^{\pi}(T)\right)\right],
$$

where $\mathbb{E}_{t}$ denotes the expectation operator conditional on the time- $t$ information. The supremum in (2) is taken over a suitable set of admissible policies, to be described in the next section, when we specialise to a market with proportional transaction costs.

Consider the alternative optimisation problem which results if a small amount of the initial wealth is diverted into the purchase or sale of a European option whose payoff at time $T$ is some non-negative random variable $C(S(T))$. To be precise, if the option price at time $t$ is $p$ and an amount of cash $\delta$ is diverted at this time into options, we define

$$
V^{(\mathrm{o})}(t, S, x-\delta, y, \delta, p)=\sup _{\pi} \mathbb{E}_{t}\left[U\left(W_{t, S, x-\delta, y}^{\pi}(T)+\frac{\delta}{P} C(S(T))\right)\right] .
$$

The "o" superscript denotes that the investor's portfolio at time $T$ incorporates the option payoff. The value function in (3) is evaluated for the initial cash endowment $x-\delta$ to signify that the funds to buy (or sell, if $\delta<0$ ) the options have come from (or been credited to) the initial wealth. The value function for initial cash $x$ is

$$
V^{(\mathrm{o})}(t, S, x, y, \delta, p)=\sup _{\pi} \mathbb{E}_{t}\left[U\left(W_{t, S, x, y}^{\pi}(T)+\frac{\delta}{P} C(S(T))\right)\right],
$$


namely the maximum utility that is achieved if the investor's account is credited with the payoff of $\delta / p$ options at time $T$, without any corresponding adjustment to the initial cash position. In (2)-(4) the quantities $\delta$ and $p$ would be measurable with respect to the time- $t$ information.

Clearly $V^{(\mathrm{o})}(t, S, x, y, 0, \cdot)=V(t, S, x, y)$, and the reader will note the dependence in (3) on the volume of option contracts traded. There are a number of ways that the above optimisation problems can be used to specify valuation bounds or a price for the option. A reservation writing price $p_{\mathrm{w}}(t, s, x, y)$ for the initial holdings $(x, y)$ is defined as the minimum value at which the investor is prepared to write the claim, and so satisfies

$$
V^{(\mathrm{o})}\left(t, S, x+p_{\mathrm{w}}(t, S, x, y), y,-p_{\mathrm{w}}(t, S, x, y), p_{\mathrm{w}}(t, S, x, y)\right)=V(t, S, x, y),
$$

since the same utility is achieved when selling the option for $p_{\mathrm{w}}(t, S, x, y)$ as is achieved by not writing the option. We further define the universal reservation writing price $\bar{p}_{\mathrm{w}}(t, S)$ as the maximum of reservation write prices across all initial holdings $(x, y)$. Therefore $\bar{p}_{\mathrm{w}}(t, S)$ satisfies

$$
V^{(\mathrm{o})}\left(t, S, x+\bar{p}_{\mathrm{w}}(t, S), y,-\bar{p}_{\mathrm{w}}(t, S), \bar{p}_{\mathrm{w}}(t, S)\right) \geqslant V(t, S, x, y) .
$$

Inequality (6) guarantees that the writer will be willing to write the option at any price higher than $\bar{p}_{\mathrm{w}}(t, S)$, independently of his current portfolio position.

Similarly, a reservation buying price $p_{\mathrm{b}}(t, S, x, y)$ for the initial holdings $(x, y)$ is

$$
V^{(\mathrm{o})}\left(t, S, x-p_{\mathrm{b}}(t, S, x, y), y, p_{\mathrm{b}}(t, S, x, y), p_{\mathrm{b}}(t, S, x, y)\right)=V(t, S, x, y) .
$$

The universal reservation buying price $p_{\mathrm{b}}(t, S)$ is the minimum of reservation buying prices across all initial holdings, and so satisfies

$$
V^{(\mathrm{o})}\left(t, S, x-\underline{p}_{\mathrm{b}}(t, S), y, \underline{p_{\mathrm{b}}}(t, S), \underline{p_{\mathrm{b}}}(t, S)\right) \geqslant V(t, S, x, y) .
$$

As pointed out by Constantinides and Zariphopoulou (1999), transaction prices of the option must lie in the interval $\left[p_{\mathrm{b}}, \bar{p}_{\mathrm{w}}\right]$. For, if an option trader writes the option for a price $p>\bar{p}_{\mathrm{w}}$ then the buyer is acting suboptimally, as he could have found a willing writer of the option at a price as low as $\bar{p}_{\mathrm{w}}$. Likewise, if a transaction occurs at a price $p<p_{\mathrm{b}}$, then the writer is acting suboptimally.

Hodges and Neuberger (1989) initiated the above approach to finding option valuation bounds. They considered the special case of a risk-neutral world where the stock return rate is equal to the riskless interest rate. In this case, and in the absence of any option transaction, the investor would choose not to invest in the stock at all. Therefore, when an option is written or purchased, the optimal trading strategy specified by (5) or (7) would consist of the incremental trades generated by the option transaction, i.e. the option hedging strategy. Hodges and Neuberger also restricted their model to the case where the agent's initial holdings in stock and cash are zero. Their method is an ingenious way of focusing attention on the hedging issue. In this paper we relax their assumptions to allow for a richer interaction between the hedging strategy and the agent's initial portfolio. Davis et al. (1993) and Constantinides and Zariphopoulou (1999) have also considered the issue of finding reservation prices for European options, without the assumption of risk-neutrality, and the latter derive analytic bounds 
on option values by embedding the pricing problem in an infinite horizon portfolio selection framework.

One way of determining a unique price for an option under market imperfections was proposed by Davis (1997). Davis suggests that an agent will be willing to trade the option at a "fair" price $\hat{p}$, such that there is a neutral effect on the investor's utility if an infinitesimal fraction of the initial wealth is diverted into the purchase or sale of the option at price $\hat{p}$. That is, $\hat{p}$ is given by the solution of

$$
\left.\frac{\partial V^{(\mathrm{o})}}{\partial \delta}(t, S, x-\delta, y, \delta, \hat{p})\right|_{\delta=0}=0 .
$$

This results in the pricing formula

$$
\hat{p}(t, S, x, y)=\frac{\mathbb{E}_{t}\left[U^{\prime}\left(W_{t, S, x, y}^{\pi^{*}}(T)\right) C(S(T))\right]}{V_{x}(t, S, x, y)},
$$

where $U^{\prime}$ is the derivative of $U, V_{x}(t, S, x, y)$ denotes the partial derivative with respect to $x$, and $\pi^{*}$ denotes the trading strategy which maximises the expected utility in (2). This is the trading strategy which optimises a portfolio without options, and the formula (10) for $\hat{p}$ shows no dependence on the optimisation problems (3) and (4) containing embedded options.

The formula in (10) is the central subject of investigation in this article. Its form is reminiscent of a classical representative agent asset pricing formula (see, for example, Duffie, 1996). Our goal is to see how (10) translates into numerical evaluations of option prices under transaction costs. It is evident from (10) that, at the price $\hat{p}$, the investor is balancing the marginal gain from diverting wealth into options with that from refraining from option trading and instead investing optimally in the stock market.

We write (10) as

$$
\hat{p}(t, S, x, y)=\frac{F(t, S, x, y)}{V_{x}(t, S, x, y)}
$$

where the function $F(t, S, x, y)$ is defined by

$$
F(t, S, x, y):=\mathbb{E}_{t}\left[U^{\prime}\left(W_{t, S, x, y}^{\pi^{*}}(T)\right) C(S(T))\right] .
$$

Because the optimal wealth vector in (12) and (2) are the same, one only needs to solve the investor's basic portfolio selection problem to calculate $F(t, S, x, y)$ and $\hat{p}(t, S, x, y)$. When transaction costs are charged on trading the stock this is a highly nontrivial simplification of the option pricing problem compared to the "reservation price" approach of (5)-(8), because the latter requires the solution of the optimisation problem with the option payoff for a large range of possible option prices.

Remark 1. In the absence of transaction costs the prices $p_{\mathrm{w}}, p_{\mathrm{b}}$ and $\hat{p}$ all reduce to the Black-Scholes option price.

\subsection{Hedging}

As well as finding sensible derivative prices under transaction costs, any feasible pricing methodology should say something concerning the risk management of an option 
position. In the case of zero transaction costs the answer to this question is automatic, in that the Black-Scholes methodology sets option prices by a hedging argument. Such comments also apply to imperfectly replicating approaches like that of Leland (1985), to quadratic, approaches like the local risk minimisation approach in Lamberton et al. (1998), and to minimisation of shortfall risk as developed by Föllmer and Leukert (2000).

In the case of a utility maximisation approach to option pricing, the situation is somewhat different, in that the pricing problem is first embedded into the utility maximisation problem to determine a price according to one of the above methods. Then one computes the optimal trading strategy in the presence of the option trade, which will be altered compared to the situation without the option, with the adjustment measuring the effect of the option trade. This adjustment will correspond to what is usually meant by an "option hedging strategy".

Suppose $\varepsilon$ options are written at price $\hat{p}$ given by (10). Then the investor's optimal trading strategy will be $\pi^{\dagger}$ maximising

$$
\mathbb{E}_{t}\left[U\left(W_{t, S, x+\varepsilon \hat{p}, y}^{\pi}(T)-\varepsilon C(S(T))\right)\right] .
$$

In the absence of the option trade, the investor's optimal trading strategy is $\pi^{*}$ to achieve the supremum in (2). Since the option trade has altered the investor's optimal stock trading strategy, a natural definition of the "option hedging strategy" is the incremental trades generated by the option trade, that is, the difference of the trading strategies $\pi^{\dagger}$ and $\pi^{*}$. This motivates the definition which follows below.

Let an amount $\delta$ be paid (or received, for the case when options are written) to trade options at time $t$ for a given price $p$. We then write the value function in (3) as

$$
V^{(\mathrm{o})}(t, S, x-\delta, y, \delta, p)=\mathbb{E}_{t}\left[U\left(W_{t, S, x-\delta, y}^{\pi^{\dagger}}(T)+\frac{\delta}{p} C(S(T))\right)\right],
$$

which defines the optimal trading strategy $\pi^{\dagger}$ for this utility maximisation problem. If we compare the optimal portfolio in the presence of the option position with that in the absence of the options, we obtain a measure of the additional holdings brought about by the option trade, which is a natural candidate for the option hedging strategy.

Definition 1. The hedging strategy $\pi^{\mathrm{h}}$ for $\delta / p$ options traded at time $t$, each at price $p$, is one whose holding $X_{t, S, x, y}^{\pi^{\mathrm{h}}}(u), Y_{t, S, x, y}^{\pi^{\mathrm{h}}}(u)$ at time $u \in[t, T]$ satisfy

$$
\begin{aligned}
& X_{t, S, x, y}^{\pi^{\mathrm{h}}}(u)=X_{t, S, x, y}^{\pi^{\dagger}}(u)-X_{t, S, x, y}^{\pi^{*}}(u), \\
& Y_{t, S, x, y}^{\pi^{\mathrm{h}}}(u)=Y_{t, S, x, y}^{\pi^{\dagger}}(u)-Y_{t, S, x, y}^{\pi^{*}}(u) .
\end{aligned}
$$

The hedging strategy can be written as $\pi^{\mathrm{h}}=\pi^{\dagger}-\pi^{*}$.

In practice, a utility maximising investor would simply calculate an optimal trading strategy $\pi^{\dagger}$ including the option trade, and would not directly calculate the hedging strategy $\pi^{\mathrm{h}}$. Nevertheless, we shall see that the above definition of a hedging strategy is a correct one, when we illustrate its features in our numerical results. 


\section{A market with transaction costs}

We consider a market consisting of a riskless bond and a risky stock whose prices $B(u)$ and $S(u)$ at time $u \in[0, T]$ satisfy, in continuous time

$$
\begin{aligned}
& \mathrm{d} B(u)=r B(u) \mathrm{d} u, \\
& \mathrm{~d} S(u)=S(u)[b \mathrm{~d} u+\sigma \mathrm{d} Z(u)],
\end{aligned}
$$

where $Z=\{Z(u), 0 \leqslant u \leqslant T\}$ is a one-dimensional standard Brownian motion defined on a complete probability space $(\Omega, \mathscr{F}, \mathbb{P})$. Denote by $\mathbb{F}=\{\mathscr{F}(u), 0 \leqslant u \leqslant T\}$ the $\mathbb{P}$-augmentation of the filtration $\mathscr{F}^{Z}(T)=\sigma(Z(u) ; 0 \leqslant u \leqslant T)$ generated by $Z$. The constant coefficients $r, b, \sigma$ represent the riskless interest rate, stock growth rate, and stock volatility, respectively. The stock is assumed to pay no dividends. Trading in the stock incurs proportional transaction costs, such that the purchase of $v$ shares of stock at price $S$ reduces the wealth held in the bond by $(1+\lambda) v S$, where $\lambda(0 \leqslant \lambda<1)$ represents the proportional transaction cost rate associated with buying stock. Similarly, the sale of $v$ shares of stock increases the wealth in the bond by $(1-\mu) v S$, where $\mu(0 \leqslant \mu<1)$ represents the proportional transaction cost rate associated with selling stock. In all other respects assume markets are "perfect". Securities are infinitely divisible, the volatility $\sigma$ is known, there is no limit on borrowing or lending at the same riskless rate, and there are no taxes or constraints on short selling with full use of the proceeds.

We shall also make use of a binomial approximation of the above market model (using a Cox et al. (1979) stock price tree, as modified by He (1990)), for numerically computing option prices and hedging strategies. The bond and stock prices follow the discrete time processes

$$
\begin{aligned}
& B(u)+\delta B(u) \equiv B(u+\delta u)=\exp (r . \delta u) B(u), \\
& S(u)+\delta S(u) \equiv S(u+\delta u)=\omega S(u),
\end{aligned}
$$

where $\omega$ is a binomial random variable:

$$
\omega=\exp \left[\left(b-\sigma^{2} / 2\right) \delta u \pm \sigma \sqrt{\delta u}\right], \quad \text { each with probability } q=\frac{1}{2},
$$

and $\delta u$ is a small time interval.

Define $(L(u), M(u))$, a pair of $\mathbb{F}$-adapted, right-continuous, non-decreasing processes, such that $L(u)$ (respectively, $M(u)$ ) is the cumulative number of shares of stock bought (respectively, sold) up to time $u$. Then in continuous time the wealth held in the bond, for an investor who begins trading in the state $(t, S, x, y)$, evolves as

$$
\mathrm{d} X(u) \equiv \mathrm{d} X_{t, S, x, y}^{L, M}(u)=r X(u) \mathrm{d} u-(1+\lambda) S(u) \mathrm{d} L(u)+(1-\mu) S(u) \mathrm{d} M(u) .
$$

The number of shares held follows the process

$$
\mathrm{d} Y(u) \equiv \mathrm{d} Y_{t, S, x, y}^{L, M}(u)=\mathrm{d} L(u)-\mathrm{d} M(u),
$$

and the wealth of the investor is given by

$$
W(u) \equiv W_{t, S, x, y}^{L, M}(u)=X_{t, S, x, y}^{L, M}(u)+Y_{t, S, x, y}^{L, M}(u) S(u) .
$$


The pair $(L, M) \equiv\{(L(u), M(u)), t \leqslant u \leqslant T\}$ constitutes a trading strategy for an investor in this financial market, who seeks to maximise expected utility of wealth at time $T$. We introduce the set $\mathscr{S}$, which defines the solvency region in the absence of an option trade, as

$$
\mathscr{S}=\left\{(S, x, y) \in \mathbb{R}^{+} \times \mathbb{R}^{2} \mid x+(1+\lambda) S y \geqslant 0, x+(1-\mu) S y \geqslant 0\right\} .
$$

A trading strategy $(L, M)$ is said to be admissible (for the problem without options) if the corresponding holdings satisfy the solvency constraint

$$
\left(S(u), X_{t, S, x, y}^{L, M}(u), Y_{t, S, x, y}^{L, M}(u)\right) \in \mathscr{S}, \quad \text { almost surely, } \forall u \in[t, T] .
$$

For an investor who trades options at time $t$ and then seeks to maximise expected utility of wealth the set of admissable trading strategies is altered. For example, when writing a contingent claim, the work of Soner et al. (1995) and Levental and Skorohod (1997) shows that, in order to keep the wealth of the writer non-negative, it is imperative to keep at least one share of the stock at all trading times. This issue does not enter our pricing methodology as it only requires us to solve an optimisation problem without the derivative security, though for computing hedging strategies this is not the case.

The value functions $V(t, S, x, y)$, and $V^{(0)}(t, S, x, y, \delta, p)$ will satisfy the same dynamic programming equations, but with different terminal boundary conditions. The function $F(t, S, x, y)$ of Eq. (12) is not necessarily a value function, but satisfies a similar recursive equation with the choice of control (the trading strategy) determined from the dynamic programming algorithm for $V(t, S, x, y)$.

The boundary equation to be applied at the terminal time $T$ for $V(t, S, x, y)$ is

$$
V(T, S, x, y)=U(x+y S)
$$

where it is assumed that there are no transaction costs charged on cashing out the final portfolio (in keeping with much of the existing literature on transaction costs). Assuming that costs are charged on liquidating the portfolio then (26) is replaced by

$$
V(T, S, x, y)=U(x+c(y, S))
$$

where $c(y, S)$ is the cash value of $y$ shares of stock, each of price $S$, and is defined by

$$
c(y, S)= \begin{cases}(1+\lambda) y S, & \text { if } y<0, \\ (1+\mu) y S, & \text { if } y \geqslant 0 .\end{cases}
$$

Our results are not qualitatively altered if there are no costs on liquidation. (The situation in a multi-asset market may be different, and we refer the reader to Kabanov (1999), who discusses hedging and liquidation of a multi-asset portfolio under transaction costs by introducing a general preference ordering for portfolios.) 
The terminal boundary condition for the optimisation problem involving options is (with the same remarks as above about liquidation costs)

$$
V^{(\mathrm{o})}(T, S, x, y, \delta, p)=U\left(x+y S+\frac{\delta}{p} C(S)\right),
$$

whilst the terminal boundary condition for $F(t, S, x, y)$ is

$$
F(T, S, x, y)=C(S) U^{\prime}(x+y S) .
$$

\subsection{Dynamic programming equations}

The dynamic programming equations satisfied by the function $V(t, S, x, y)$ in a market with proportional transaction costs have been derived by Hodges and Neuberger (1989) and Davis et al. (1993). We present these equations below along with a simple (and financially intuitive) justification, and we describe the investor's optimal trading strategy. We highlight how the characteristics of the value function $V(t, S, x, y)$ and the associated optimal portfolio strategy impinge on the investor's option valuation and hedging policy.

The investor's optimal strategy at any state $(t, S, x, y)$ is as follows. The state space $(t, S, x, y)$ is split into three distinct regions: the BUY, SELL and no transaction (NT) regions, from which it is optimal to buy stock, sell stock and not to trade, respectively. Moreover, with proportional transaction costs, the optimal trade when outside the NT region is to transact to the nearest boundary of the NT region. The optimal strategy can be summarised as minimal trading to keep the portfolio in the NT region. We denote the boundaries between the NT region and the BUY and SELL regions by $y_{\mathrm{b}}$ and $y_{\mathrm{s}}$, respectively. In general, $y_{\mathrm{b}}$ and $y_{\mathrm{s}}$ will be functions of $(t, S, x)$, and will represent the number of shares held at the NT boundaries. If the state is in the NT region it drifts under the influence of the diffusion driving the stock price, on a surface defined by $Y(u)=$ constant. If the state is in the BUY or SELL regions, an immediate transaction occurs taking the state to the nearest boundary of the NT region.

In the BUY region the value function remains constant along the path of the state dictated by the optimal trading strategy, and therefore satisfies

$$
V(t, S, x, y)=V(t, S, x-S(1+\lambda) \delta L, y+\delta L) \quad \text { in BUY, }
$$

where $\delta L$, the number of shares bought, can take any positive value up to the one required to take the portfolio to $y_{\mathrm{b}}$. Allowing $\delta L \downarrow 0$, (31) becomes

$$
\frac{\partial V}{\partial y}(t, S, x, y)-(1+\lambda) S \frac{\partial V}{\partial x}(t, S, x, y)=0 \quad \text { in BUY. }
$$

Eq. (32) indicates that in the BUY region the marginal utilities of the risky and riskless assets are in the ratio $(1+\lambda) S$, the cost of purchasing one share of the stock. It defines a direction in the state space in which the value function is constant in the BUY region. Such properties of the value function will carry over to the investor's option valuation and hedging strategy. Specifically, the general option pricing formula (10) yields an option price which does not vary with the investor's initial portfolio outside the NT region. 
Similarly, in the SELL region, the value function satisfies

$$
V(t, S, x, y)=V(t, S, x+S(1-\mu) \delta M, y-\delta M) \quad \text { in SELL, }
$$

where $\delta M$ represents the number of shares sold. Letting $\delta M \downarrow 0$, (33) becomes

$$
\frac{\partial V}{\partial y}(t, S, x, y)-(1-\mu) S \frac{\partial V}{\partial x}(t, S, x, y)=0 \quad \text { in SELL. }
$$

Eqs. (32) and (34) are the well known "value matching" conditions satisfied by the value function of (2). (See, for example, Dixit (1991), Dumas (1991, 1992), Dumas and Luciano (1991), or the exposition by Harrison (1985) of the theory of optimally regulated Brownian motion.) They hold throughout the BUY and SELL regions, and (of course) at the boundaries $y_{\mathrm{b}}, y_{\mathrm{s}}$, regardless of whether these are chosen optimally or not.

Finally, in the NT region, since it is sub-optimal to carry out any stock trades, for any stock purchase $\delta L$ or sale $\delta M$ :

$$
V(t, S, x, y) \geqslant V(t, S, x-S(1+\lambda) \delta L, y+\delta L) \quad \text { in } \mathrm{NT}
$$

and

$$
V(t, S, x, y) \geqslant V(t, S, x+S(1-\mu) \delta M, y-\delta M), \quad \text { in NT, }
$$

which on expansion imply that the left hand sides of (32) and (34) are non-positive and non-negative, respectively, in NT. Bellman's optimality principle for dynamic programming gives the value function at time $t$ in terms of its counterpart at time $t+\delta t$ as

$$
V(t, S, x, y)=\mathbb{E}_{\delta t} V(t+\delta t, S+\delta S, x+\delta x, y) \quad \text { in } \mathrm{NT},
$$

where $\mathbb{E}_{\delta t}$ denotes expectation over the time interval $\delta t$. In the limit $\delta t \rightarrow 0, \delta S$ and $\delta x$ are given by (17) and (21), respectively (with $\mathrm{d} L=\mathrm{d} M=0$ since we are in the NT region). Applying Itô's lemma yields the Hamilton-Jacobi-Bellman equation for the value function in the NT region:

$$
V_{t}+r x V_{x}+b S V_{S}+\frac{1}{2} \sigma^{2} S^{2} V_{S S}=0 \quad \text { in } \mathrm{NT},
$$

where the arguments of the value function have been omitted for brevity.

These equations can be condensed into the PDE

$$
\max \left[V_{y}-(1+\lambda) S V_{x},-\left(V_{y}-(1-\mu) S V_{x}, V_{t}+r x V_{x}+b S V_{s}+\frac{1}{2} \sigma^{2} S^{2} V_{S S}\right]=0 .\right.
$$

The solution of the optimisation problem is obtained by observing that if we can compute the value function in the NT region along with the boundaries of this region, then we can calculate its value in the BUY and SELL regions using (31) and (33).

A closed form solution for the value function $V(t, S, x, y)$ is elusive. This feature is (notoriously) common to models with transaction costs. Constantinides (1986), Davis and Norman (1990), Dumas and Luciano (1991) and Shreve and Soner (1994) do find, in the infinite-horizon (and hence, time independent) case, for HARA utility functions, an analytic form for the value function. However, the boundaries of the NT region 
must still be located numerically. We shall, therefore, in our finite-horizon problem, go directly to the development of a numerical dynamic programming algorithm to obtain option prices and hedging strategies.

We use Eqs. (31), (33) and (37), and augment them with the specific properties of the optimal trading strategy to create a backward recursive dynamic programming algorithm. This yields the value function at time $t$, provided it is known at time $t+\delta t$, along with the location of the NT boundaries. Assume that the stock and bond prices evolve in discrete time according to (18)-(20). Then the discrete dynamic programming equation is

$$
\begin{aligned}
V(t, S, x, y)= & \max _{(\delta L, \delta M)}\left[\mathbb{E}_{\delta t} V(t+\delta t, \omega S, R(x-S(1+\lambda) \delta L), y+\delta L),\right. \\
& \mathbb{E}_{\delta t} V(t+\delta t, \omega S, R x, y), \\
& \left.\mathbb{E}_{\delta t} V(t+\delta t, \omega S, R(x+S(1-\mu) \delta M), y-\delta M)\right],
\end{aligned}
$$

where $R=\exp (r . \delta t)$ and the maximum is achieved by the first, second or third terms in (40) when the state $(t, S, x, y)$ is in the BUY, NT and SELL regions, respectively.

Eq. (40) expresses the value function at time $t$ in terms of its counterpart at $t+\delta t$ by comparing the three possibilities: (i) buying $\delta L$ shares and allowing the stock to diffuse or (ii) not trading and allowing the stock to diffuse or (iii) selling $\delta M$ shares and allowing the stock to diffuse.

The algorithm is an example of the Markov chain approximation technique for the numerical solution of continuous time stochastic control problems, pioneered by Kushner (1990); see also Kushner (1997) for a review of applications in finance. The state variables and controls are approximated by discrete-time, discrete-state Markov chains, in such a manner that the solution to the discrete problem converges to the solution of the continuous-time problem. The application here is to a singular control problem, along the lines of Kushner and Martins (1991). For the optimal portfolio problem studied here, the necessary proofs of convergence of the discrete time problem to the continuous one are supplied by Davis et al. (1993), who proved that the value function $V(t, S, x, y)$ is a viscosity solution of the variational inequality (39).

To implement the above algorithm we specialise to the case of exponential utility in the next section.

\section{Option prices and hedging strategies under exponential utility}

Following Hodges and Neuberger (1989) and Davis et al. (1993) we set the investor's utility function to be the negative exponential:

$$
U(W)=-\exp (-\alpha W)
$$

with constant risk aversion parameter $\alpha$. With this choice the investor's optimal trading strategy becomes independent of the wealth held in the riskless asset. The assumption of exponential utility is made primarily to reduce the dimensionality of the optimisation problems that we must solve, as our goal is to compute numerical estimates 
of the option prices and hedging strategies implied by the general option pricing formula (10). We defer to a later article the comparison of option prices generated by alternative choices of the utility function. For example, with HARA utility functions, such as logarithmic or power utility, the optimal trading strategy is characterised by a time-varying no-transaction region with boundaries $\zeta_{\mathrm{b}}(t)<\zeta_{\mathrm{s}}(t)$, where $\zeta(t)$ represents the ratio of wealth held in the stock to that held in the bond at time $t$. We hypothesise that the results for option prices will be similar to those we present below for exponential utility, but the relevant state-space variable will be $\zeta(t)$ instead of the amount of money invested in the stock.

Below we show how the optimisation problem for $V(t, S, x, y)$ simplifies under exponential utility.

Define

$$
H(t, S, y):=V(t, S, 0, y)
$$

then since, with exponential utility, the optimal portfolio through time is independent of the wealth held in the bond, we have that

$$
V(t, S, x, y)=H(t, S, y) \exp \left(-\alpha x e^{r(T-t)}\right) .
$$

The resulting reduction in dimensionality means that the discrete dynamic programming algorithm (40) reduces to

$$
\begin{aligned}
H(t, S, y)= & \max _{(\delta L, \delta M)}\left[\mathbb{E}_{\delta t} H(t+\delta t, \omega S, y+\delta L) \exp (\alpha S(1+\lambda) \delta L . \beta(t)),\right. \\
& \mathbb{E}_{\delta t} H(t+\delta t, \omega S, y), \\
& \left.\mathbb{E}_{\delta t} H(t+\delta t, \omega S, y-\delta M) \exp (-\alpha S(1-\mu) \delta M . \beta(t))\right],
\end{aligned}
$$

where $\beta(t)=\exp (r(T-t))$.

For exponential utility, the boundaries of the NT region become functions of $t$ and $S$ only. Denote them by $y_{\mathrm{b}}(t, S)$ and $y_{\mathrm{s}}(t, S)$, with $y_{\mathrm{b}}(t, S) \leqslant y_{\mathrm{s}}(t, S)$, and equality holding only in the case where $\lambda=\mu=0$.

The optimal values of $\delta L$ and $\delta M, \delta L^{*}$ and $\delta M^{*}$ satisfy

$$
\begin{gathered}
y+\delta L^{*}=y_{\mathrm{b}}(t, S) \text { and } \delta M^{*}=0 \quad \text { if } y<y_{\mathrm{b}}(t, S) \\
\delta L^{*}=\delta M^{*}=0 \quad \text { if } y_{\mathrm{b}}(t, S) \leqslant y \leqslant y_{\mathrm{s}}(t, S) \\
\delta L^{*}=0 \text { and } y-\delta M^{*}=y_{\mathrm{s}}(t, S) \quad \text { if } y>y_{\mathrm{s}}(t, S) .
\end{gathered}
$$

Applying (45) and (43) to Eqs. (31), (33) and (37), we also obtain the following representation for $H(t, S, y)$ in the BUY, SELL and NT regions.

If $y<y_{\mathrm{b}}(t, S)$, then

$$
H(t, S, y)=H\left(t, S, y_{\mathrm{b}}(t, S)\right) \exp \left(\alpha S(1+\lambda)\left(y_{\mathrm{b}}(t, S)-y\right) \beta(t)\right) .
$$

If $y>y_{\mathrm{s}}(t, S)$, then

$$
H(t, S, y)=H\left(t, S, y_{\mathrm{s}}(t, S)\right) \exp \left(-\alpha S(1-\mu)\left(y-y_{\mathrm{s}}(t, S)\right) \beta(t)\right) .
$$


If $y_{\mathrm{b}}(t, S) \leqslant y \leqslant y_{\mathrm{s}}(t, S)$, then

$$
H(t, S, y)=\mathbb{E}_{\delta t} H(t+\delta t, \omega S, y)
$$

Eqs. (46)-(48) give the value function $H(t, S, y)$ in the BUY, NT and SELL regions, provided we know $H(t, S, y)$ at and within the boundaries $y_{\mathrm{b}}(t, S)$ and $y_{\mathrm{s}}(t, S)$, along with the location of these boundaries. These are located by implementing the algorithm in (44) in the manner described below.

We create a large vector to represent possible values of $y$ at each node of the stock-price tree, with discretisation step $h_{y}$. The range of this vector must be large enough to locate $y_{\mathrm{b}}(t, S)$ and $y_{\mathrm{s}}(t, S)$ for all $(t, S)$ on the binomial stock-price tree. This can be accomplished by deriving analytically the NT boundaries at $T-\delta t$ (see Appendix A for this derivation), and noting that the NT region is wider at this time than at any preceding time. Then the following sequence of steps is performed.

1. Suppose we know the value function at $t+\delta t$ for all stock prices on the binomial tree at this time, and for all values of $y$ in our discrete vector. Then starting at a time- $t$ node of the stock-price tree, $(t, S)$ say, and from the minimum value of $y$ in this vector, we compare the first and second terms in the maximisation operator of (44) for increasing values of $y$ in steps of $h_{y}$, until the latter is greater than or equal to the former, at say $y^{\mathrm{b}}$, which we assume satisfies $y^{\mathrm{b}}=y_{\mathrm{b}}(t, S)$, the boundary between the NT and BUY regions at the node $(t, S)$.

2. We continue, comparing the second and third terms in the maximisation operator of (44) for increasing values of $y$ in steps of $h_{y}$, until the latter is greater than or equal to the former, at say $y^{\mathrm{s}}$, which we assume satisfies $y^{\mathrm{s}}=y_{\mathrm{s}}(t, S)$, the boundary between the NT and SELL regions at the node $(t, S)$.

3. Having located the boundaries of the NT region at the node $(t, S)$, the value function at all points outside this region is determined by assuming the investor transacts to its boundaries (i.e. applying Eqs. (46) and (47)), whilst the function in the NT region is found by assuming the investor does not transact, and applying Eq. (48).

We further enhance the speed of the above algorithm for $V(t, S, x, y)$ because, for exponential utility, the NT boundaries at any time are characterised by the wealth held in the stock being constant. (This was confirmed by solving the problem without this assumption.) Therefore, having located the boundaries at a single node of the binomial tree at time $t$, the boundaries at all other time- $t$ nodes are given easily. This property is not satisfied by the value function $V^{(0)}(t, S, x, y, \delta, p)$.

The other noteworthy feature of our algorithm is the fact that the $y$-vector we use is bounded to the interval $\left[y_{\mathrm{b}}(T-\delta t, S), y_{\mathrm{s}}(T-\delta t, S)\right]$, the NT boundaries one period prior to the option expiry. This allows us to restrict the state space over which we carry out the search for the NT boundaries at all earlier times, and makes for fast, accurate computation. These features can be exploited for option pricing because the "fair" pricing methodology only requires the solution of the investor's optimal portfolio problem in the absence of options. 


\subsection{Option prices}

To calculate option prices, under exponential utility, the fair pricing formula (11) becomes

$$
\hat{p}(t, S, y)=\mathrm{e}^{-r(T-t)} \frac{G(t, S, y)}{H(t, S, y)},
$$

where

$$
G(t, S, y)=\mathbb{E}_{t}\left[U\left(W_{t, S, 0, y}^{\pi^{*}}(T)\right) C(S(T))\right],
$$

using the fact that $U^{\prime}(w)=-\alpha U(w)$.

\subsection{Effect of option trading on utility}

To compare the investor's maximum utility with and without the diversion of funds into an option trade, we must compare, in general, the quantities $V^{(\mathrm{o})}(t, S, x-\delta, y, \delta, p)$ with $V(t, S, x, y)$. With exponential utility this becomes a comparison of

$$
H^{(o)}(t, S, y, \delta, p) \exp (\alpha \delta \beta(t)) \quad \text { with } H(t, S, y),
$$

where $H^{(o)}(t, S, y, \delta, p)$ is defined by

$$
H^{(\mathrm{o})}(t, S, y, \delta, P) \equiv V^{(\mathrm{o})}(t, S, 0, y, \delta, p) .
$$

\section{Numerical results}

For our numerical results we used the following parameters as a base case: $T=1$ year, $r=0.1, b=0.15, \sigma=0.25, \alpha=0.1$, and we took the transaction cost rates for buying and selling stock to be equal $(\mu=\lambda)$. We used a stock-price tree with at least 50 timesteps. First we confirmed some stylised facts about the investor's optimal trading strategy without options, which we summarise below, and which verify the robustness of our numerical algorithm. The optimal trading strategy has the following properties.

1. The boundaries of the NT region lie either side of the optimal portfolio without transaction costs, and the NT region widens with the transaction costs.

2. The NT region boundaries show a hyperbolic dependence versus the stock price, just as in the frictionless markets case, indicating that with exponential utility, and at a fixed time, the wealth in the stock is constant at the boundaries of the NT region.

3. As we approach the horizon time $T$ the NT region widens considerably. This is as expected, indicating that portfolio rebalancing becomes less advantageous as the time to expiry lessens. This feature carries over to the hedging strategy for an option position.

4. An increase in risk aversion narrows the region of no transactions and shifts it to lower values of the stock holding. 


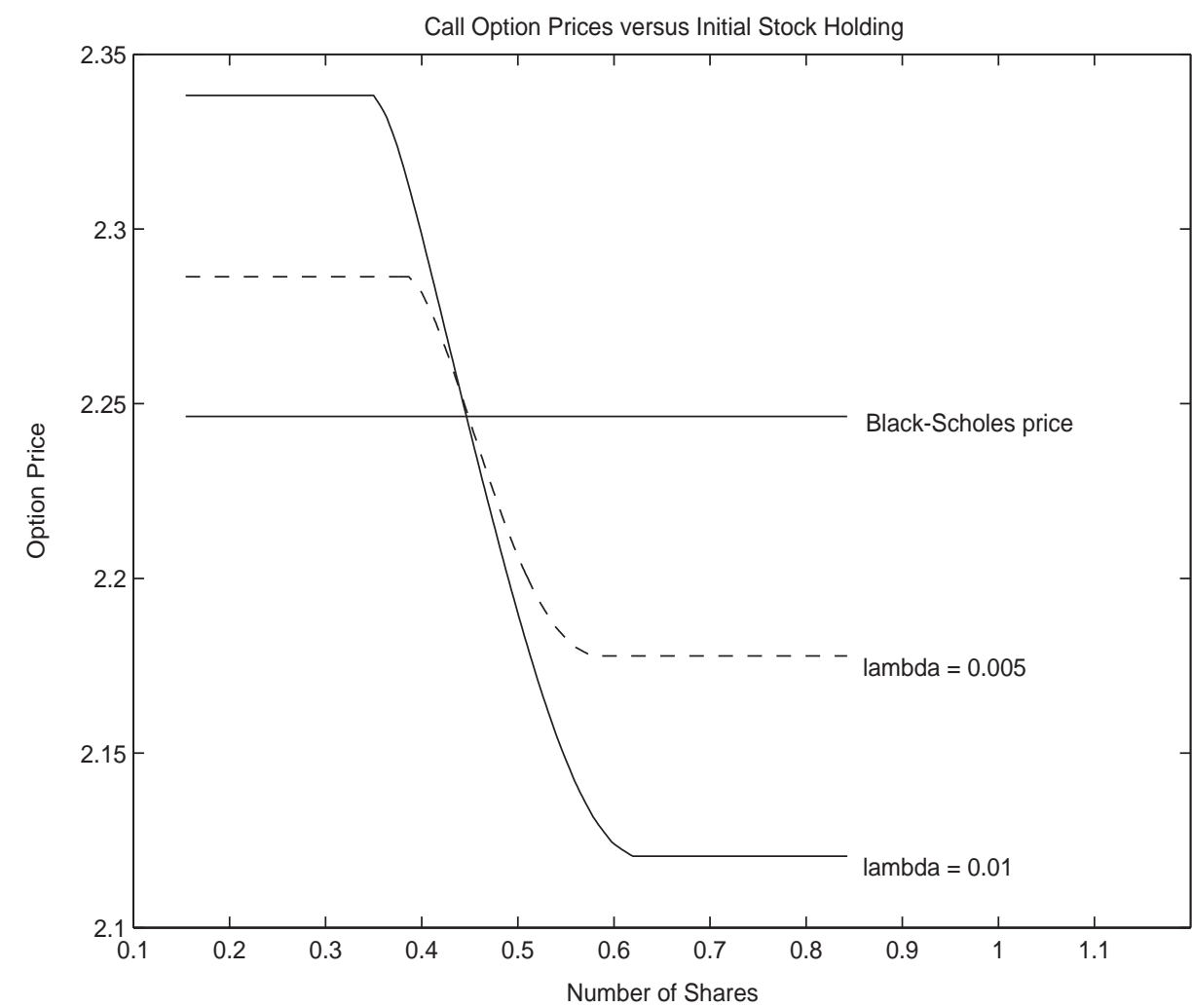

Fig. 1. At-the-money call option prices versus initial stock holding. The parameters are $T=1$ year, $r=0.1$, $b=0.15, \sigma=0.25, \alpha=0.1, S=K=15, \lambda=0.01$ and 0.005 .

Fig. 1 shows at-the-money call option prices given by the general option pricing formula (49), plotted at time zero versus the investor's initial stock holding, $y$, for transaction cost parameters $\lambda=0.005$ and $\lambda=0.01$. The graphs are flat outside a certain range of $y$, which corresponds exactly with the width of the NT region for the particular transaction cost parameter. We see the widening of the option pricing bounds as the transaction costs are increased. The fair option price is higher when the investor's stock inventory is in the BUY region for the basic portfolio selection problem, then falls as we enter the NT region, and is at its lowest when the current inventory position is in the investor's SELL region. This is intuitively correct, since a buyer of shares will value a call option more highly than someone who wishes to sell stock. Of course, the opposite pattern is obtained for put options.

\subsection{The effect on utility of trading options}

We analyse the case of the investor writing a call option at price $p_{\mathrm{w}}$, the maximum price in Fig. 1, which we label the "fair asking price". If we compare $H^{(\mathrm{o})}(t, S, n$, 
$\left.-p_{\mathrm{w}}, p_{\mathrm{w}}\right) \exp \left(-\alpha p_{\mathrm{w}} \beta(t)\right)$ with $H(t, S, y)$ (making the comparison in (51) with $\delta=-p_{\mathrm{w}}$ and $p=p_{\mathrm{w}}$ ), we find that the investor's utility is virtually unchanged by the option sale. However, trading an option at a price outside the bid-ask spread always results in a reduction in utility (if one buys above the asking price or sells below the bid price) or an increase in utility (if one sells above the asking price or buys below the bid price).

To quantify the above points, in Fig. 2 (upper graph) we have plotted the difference between the investor's maximum utility when selling one call option at the fair asking price and the maximum utility in the absence of the option sale. If the investor starts out with a low stock inventory (i.e. he would, acting optimally, buy shares) then selling a call option reduces his utility (albeit by a small amount). At some value, $y^{*}$, of the initial stock holding $y$, the investor finds his utility is unchanged by the diversion of funds into the option transaction, and for $y>y^{*}$ the utility is increased by the sale of the option. The increase in utility reaches a maximum, then tails off toward zero for large values of $y$, reflecting the fact that when the investor wishes to sell large amounts of stock, selling a call option increases utility, but this effect diminishes as the initial stock holding increases. For all values of $y$, the percentage change in utility is bounded and small, as shown in the lower graph. Similar observations hold for any trade of an option within the "fair" bid-ask spread.

To summarise, the fair price given by the general option pricing formula is essentially a reservation price. A nice feature of the pricing method used in this paper is that the option pricing bounds can be found by solving the investor's basic portfolio selection problem, and without having to solve the more complicated problem involving the purchase or sale of options.

Fig. 3 shows at the money call option prices for two different risk aversion parameters. The bid-ask spread is independent of risk aversion, but the range of values of the initial stock holding for which the fair price lies within the bid-ask spread becomes narrower and is shifted to a lower value, as the risk aversion increases.

In Table 1 we present call option prices for various strikes and transaction cost parameters, and for comparison we show the bid and ask prices generated by Leland's (1985) approximately replicating strategy, with a revision interval of $\delta t=0.02$, which corresponds to approximately weekly portfolio rebalancing. A number of points are worth emphasising. First, in general, the optimal pricing approach places tighter bounds on the option price (except for options that are deep in-the-money, the intuition for which is given below), particularly for large transaction costs. The intuition behind this feature is natural: Leland's strategy insists on portfolio rebalancing (thus incurring transaction costs) in situations where the optimal pricing procedure may not. We used a binomial tree with the same time step as the Leland revision interval to generate the prices in Table 1, which means that the investor has the opportunity to rehedge as frequently as the Leland strategy, but chooses not to do so.

The implication of these results is that the investor is prepared to bear more risk than the Leland strategy allows, and the size of this risk is determined by the utility function. The only exception to this feature is for options which are deep in-the-money. In this case Leland's bounds are tighter. The intuition here is as follows: for a deep in the money option, with very high probability of exercise, the optimal policy is 

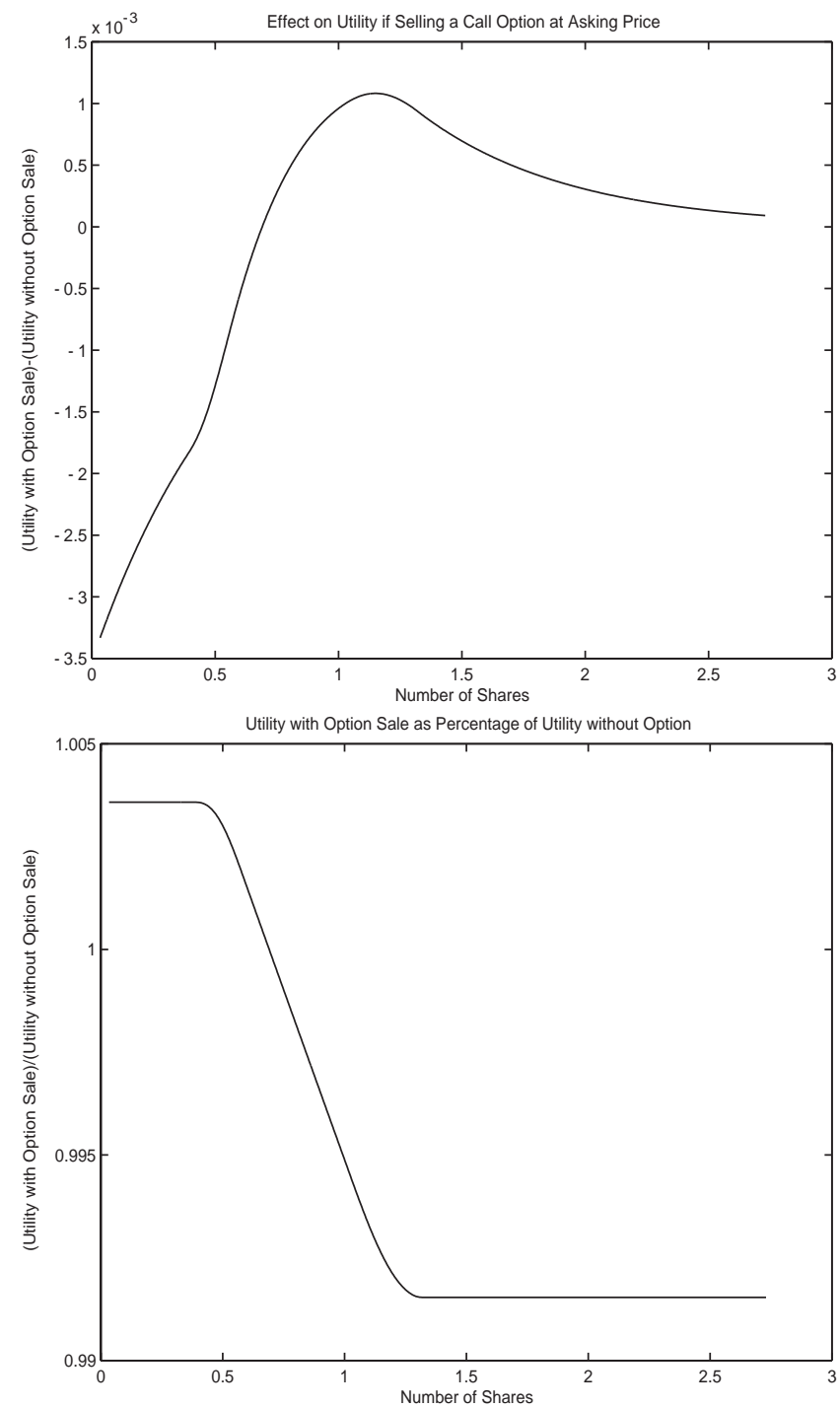

Fig. 2. The upper graph shows the difference between the investor's maximum utility when selling a call option at the "fair" asking price and the maximum utility without selling the option. The lower graph plots the utility with the option sale as a fraction of the utility without the option sale. The parameters are $T=1$ year, $r=0.1, b=0.15, \sigma=0.25, \alpha=0.1, S=K=15, \lambda=0.005$ and $\hat{p}=p_{\mathrm{w}}=2.2864$.

to be (almost) fully hedged, and this is in accordance with Leland's strategy, which is designed to eliminate risk in a Black-Scholes type manner. Therefore, in these situations, Leland's strategy is optimal and falls within the spread given by utility maximisation. 

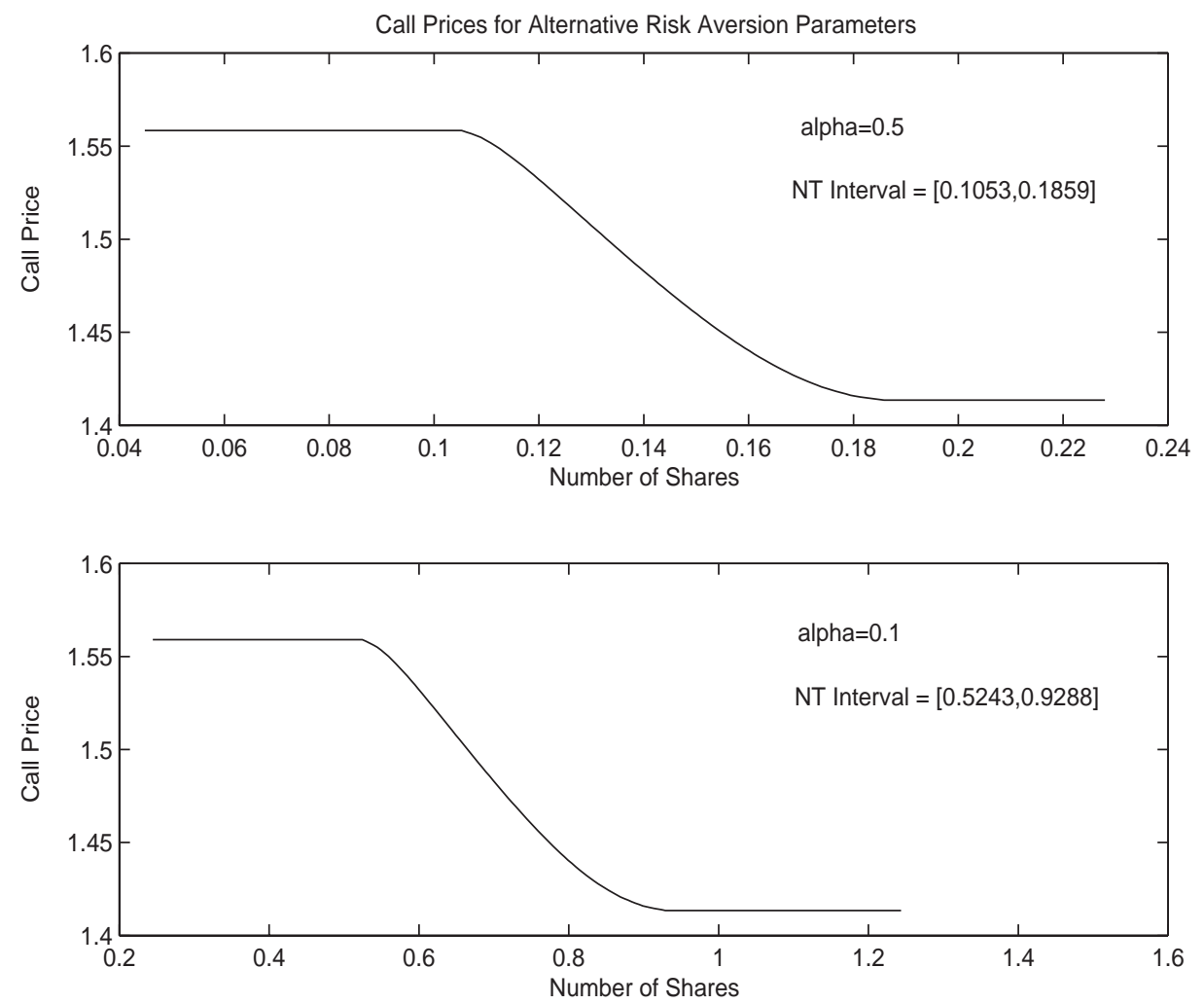

Fig. 3. Call option prices for different risk aversion parameters. The parameters are $T=1$ year, $r=0.1$, $b=0.15, \sigma=0.25, S=K=10, \lambda=0.01, \alpha=0.5$ and 0.1 .

For very large transaction costs Leland's strategy fails to produce a bid price for the option, as the effective volatility is no longer a real number. This point has also been made by Avellaneda and Parás (1994), who provided a solution to this problem using the notion of imperfectly dominating policies. The optimal pricing procedure never fails to produce a sensible option price, regardless of the level of transaction costs.

Fig. 4 shows a plot of the call bid-ask spread specified by the optimal pricing formula, versus the stock price. We have also shown Leland's bid-ask spread with a revision interval $\delta t=0.02$, equal to the time step of the binomial tree, and the BlackScholes call values. We see how the optimal pricing procedure places tighter bounds on the option prices, except for the cases where the option is deep in the money, as before.

In Fig. 5 we plot the hedging strategy for a short call position versus the initial stock price, produced-using Definition 1. The dashed curves indicate the region in which the hedging portfolio is not rebalanced, whilst the solid curve is the BlackScholes delta hedging strategy. The replacement of the unique Black-Scholes delta by a hedging bandwidth is in accordance with intuition and with previous results on 
Table 1

Call bid and ask prices

\begin{tabular}{|c|c|c|c|c|}
\hline Strike & Ask Price & Bid Price & Leland Ask & Leland Bid \\
\hline \multicolumn{5}{|c|}{$\lambda=0.005$, NT Region $=[0.3866,0.5780]$} \\
\hline 10 & 6.0471 & 5.8980 & 5.9940 & 5.9610 \\
\hline 13 & 3.5841 & 3.4503 & 3.6190 & 3.4348 \\
\hline 15 & 2.2864 & 2.1775 & 2.3869 & 2.0915 \\
\hline 17 & 1.3419 & 1.2641 & 1.4878 & 1.1481 \\
\hline 20 & 0.5423 & 0.5048 & 0.6724 & 0.3949 \\
\hline \multicolumn{5}{|c|}{$\lambda=0.01$, NT Region $=[0.3499,0.6197]$} \\
\hline 10 & 6.1199 & 5.8248 & 6.0187 & 5.9537 \\
\hline 13 & 3.6476 & 3.3837 & 3.7088 & 3.3458 \\
\hline 15 & 2.3376 & 2.1212 & 2.5164 & 1.9171 \\
\hline 17 & 1.3788 & 1.2210 & 1.6336 & 0.9398 \\
\hline 20 & 0.5613 & 0.4805 & 0.8010 & 0.2470 \\
\hline \multicolumn{5}{|c|}{$\lambda=0.02$, NT Region $=[0.2702,0.7196]$} \\
\hline 10 & 6.2675 & 5.6716 & 6.0775 & 5.9516 \\
\hline 13 & 3.7798 & 3.2463 & 3.8807 & 3.2374 \\
\hline 15 & 2.4475 & 2.0073 & 2.7502 & 1.4800 \\
\hline 17 & 1.4612 & 1.1361 & 1.8940 & 0.3057 \\
\hline 20 & 0.6063 & 0.4348 & 1.0401 & 0.0034 \\
\hline \multicolumn{5}{|c|}{$\lambda=0.03$, NT Region $=[0.1813,0.8243]$} \\
\hline 10 & 6.4068 & 5.5242 & 6.1450 & - \\
\hline 13 & 3.9070 & 3.1159 & 4.0421 & - \\
\hline 15 & 2.5556 & 1.9012 & 2.9590 & - \\
\hline 17 & 1.5445 & 1.0589 & 2.1242 & - \\
\hline 20 & 0.6537 & 0.3948 & 1.2592 & - \\
\hline
\end{tabular}

The parameters are $T=1$ year, $r=0.1, b=0.15, \sigma=0.25, \alpha=0.1, S=15$. For $\lambda=0.03$ the Leland bid price is undefined for a revision interval of $\delta t=0.02$.

optimal hedging under transaction costs, such as Hodges and Neuberger (1989) and (for the limiting case of small transaction costs) Whalley and Wilmott (1997).

\section{Conclusions and suggestions}

This paper has developed a procedure for optimally valuing options in the presence of proportional transaction costs. The method involves treating an option transaction as an alternative investment to optimally trading the underlying stock. Option prices are determined by requiring that, at the margin, the diversion of funds into an option trade has no effect on an investor's achievable utility. Thus, the option trade is treated as a small perturbation on the investor's initial portfolio of assets. The methodology can therefore be extended to situations in which the basic portfolio contains many assets, including possibly other derivatives. 


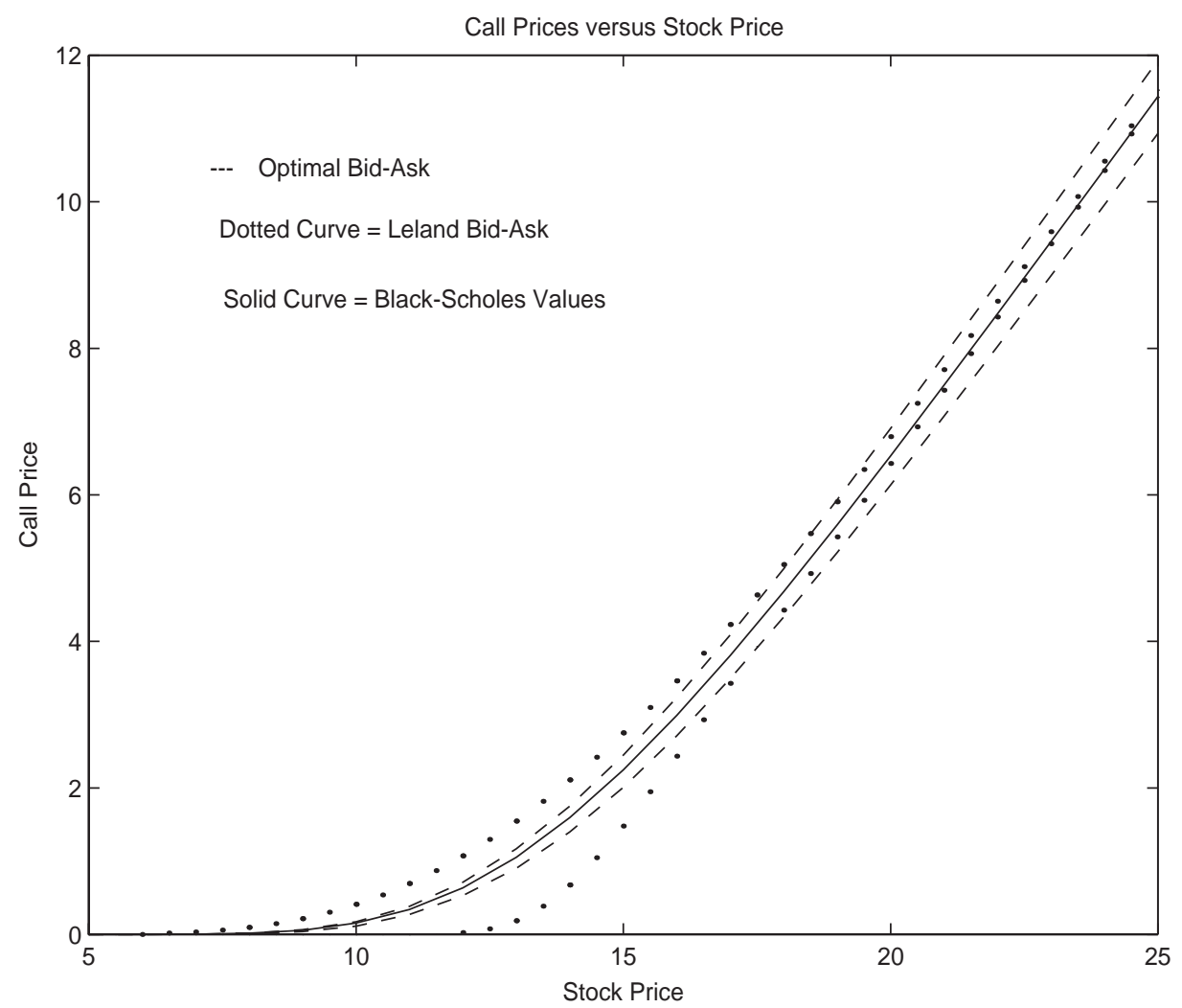

Fig. 4. Call option bid-ask values versus stock price. The parameters are $T=1$ year, $r=0.1, b=0.15$, $\sigma=0.25, \alpha=0.1, K=15, \lambda=0.02$.

Option prices are computed by solving a singular stochastic optimal control problem via an efficient algorithm. We only need to solve the investor's fundamental portfolio selection problem to derive option prices, as opposed to other optimal procedures which require the solution of an optimisation problem containing an embedded option. The method is therefore a relatively tractable way of producing optimal pricing bounds.

The method places tight bounds on option prices and generates prices which can lie anywhere within these bounds, depending on the investor's initial holding of stock. The investor's utility is hardly affected when trading options at the optimal prices, whereas trading outside the bounds can lead to a drastic loss in utility.

The final conclusion is that a general approach to option pricing in the presence of transaction costs should be based on an optimal portfolio approach. Approximate replication schemes work well for small levels of transaction costs and are useful because they can lead to closed form approximate solutions, but they cannot cope with larger values of transaction costs. In these cases the optimal models outperform them as the frequency of trading is reduced. 


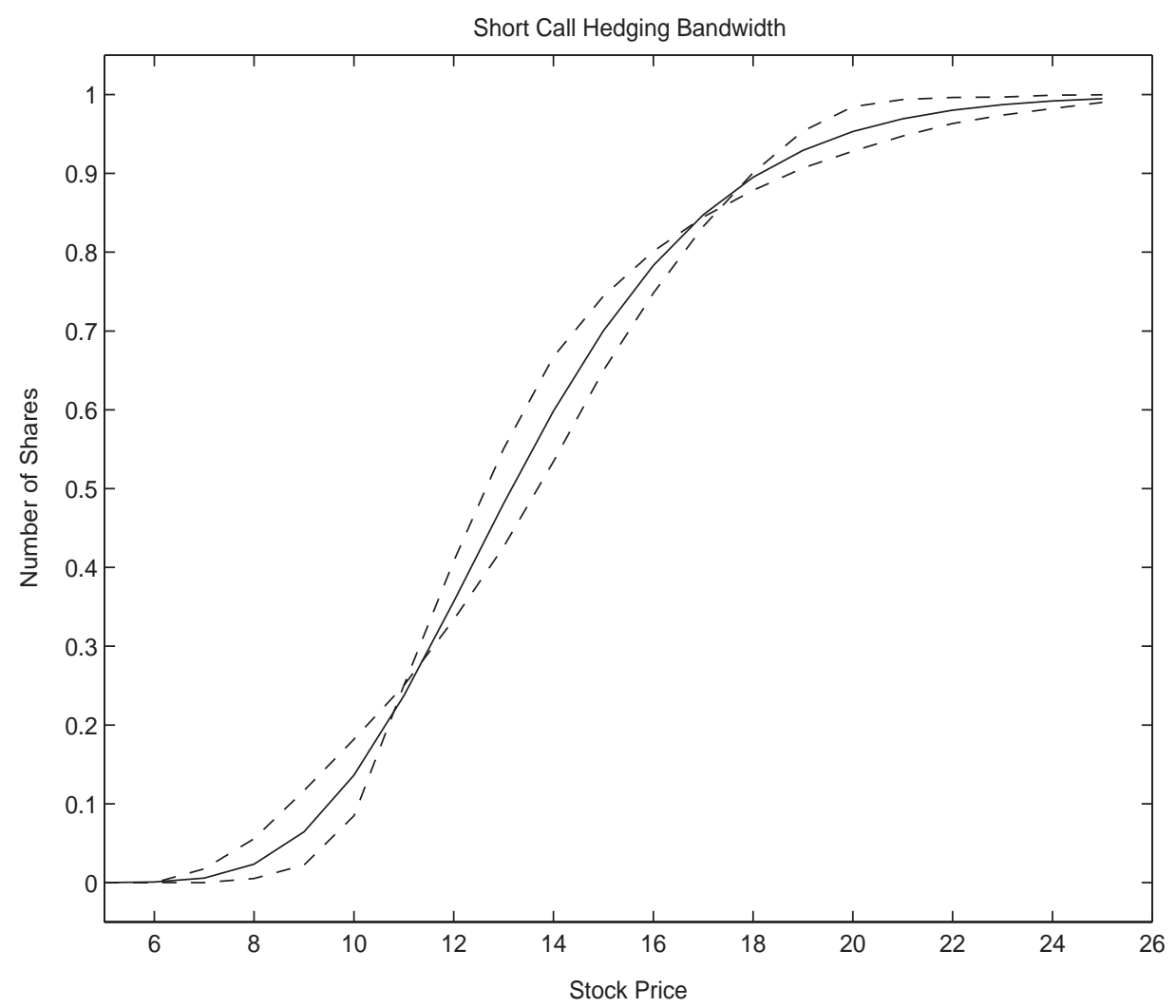

Fig. 5. Short call option hedging strategies versus stock price. The call was sold at the "fair" asking price, and the other parameters are $T=1$ year, $r=0.1, b=0.15, \sigma=0.25, \alpha=0.1, K=15, \lambda=0.005$. The dashed curve indicates the region in which portfolio rebalancing does not take place, whilst the solid curve is the Black-Scholes delta.

There are a number of directions in which this work could be extended:

American options. The pricing of American options with transaction costs presents some interesting problems because one not only has to compute an optimal hedging strategy, but also an optimal exercise policy. This will involve a problem in singular control with optimal stopping, which has been studied by Davis and Zervos (1994). There is a further complication for the writer of an option in that it is not he, but the buyer of the option, who controls the exercise policy. Some preliminary ideas on this topic have been provided by Davis and Zariphopoulou (1995).

Equilibrium. The option pricing method described in this paper selects a "fair" price within a closed interval provided by the reservation buying and selling prices. It therefore gives some hope of providing a framework in which the buyer and seller of an option might agree on a price.

Different preferences. An analysis of how the risk preferences of the investor affect option prices would be interesting. Optimal portfolios for HARA utility functions are 
usually determined by selecting an optimal ratio of wealth in the risky and riskless assets, as opposed to the exponential function used in this paper, in which the wealth held in the risky asset is the important variable. We hypothesise that such patterns would transfer to the option valuation problem. One could also consider quadratic preferences, such as risk minimisation, as in Mercurio and Vorst (1997) or Lamberton et al. (1998). A further possibility is to consider "coherent" measures of risk, introduced by Artzner et al. (1999), and extended to a dynamic setting by and Cvitanic and Karatzas (1999). One example of such a risk measure is provided by the "shortfall risk" minimisation of Föllmer and Leukert (2000). In general, the interaction between agents' preferences and option prices in incomplete markets is a fertile area for future research, and recent papers addressing this topic include Rouge and El Karoui (2000), Barrieu and El Karoui (2002) and Musiela and Zariphopoulou (2001).

\section{Acknowledgements}

The author wishes to thank Bill Ziemba and three anonymous referees, for helpful comments.

\section{Appendix A}

We derive analytic formulae for the value function $H(T-\delta t, S, y)$ and the boundaries of the NT region $y_{\mathrm{b}}(T-\delta t, S), y_{\mathrm{s}}(T-\delta t, S)$, one time period prior to the final time $T$, under exponential utility.

In the BUY region $\left(y<y_{\mathrm{b}}(T-\delta t, S)\right)$ the Bellman equation (44) for the value function $H(T-\delta t, S, y)$ reduces to

$$
H(T-\delta t, S, y)=\max _{\delta L} \mathbb{E}_{\delta t} H(T, \omega S, y+\delta L) \exp (\alpha R S(1+\lambda) \delta L), \quad \text { in BUY }
$$

where $R=\exp (r . \delta t)$ and $H(T, S, y)=-\exp (-\alpha y S)$.

We write out the above expectation explicitly, differentiate with respect to $\delta L$, and set the result to zero. This yields, after some tedious algebra, that the optimal number of shares to buy, $\delta L^{*}$, satisfies

$$
y+\delta L^{*} \equiv y_{\mathrm{b}}(T-\delta t, S)=\frac{1}{\alpha S\left(\omega_{u}-\omega_{d}\right)} \log \left(\frac{q\left(1-q_{+}\right)}{(1-q) q_{+}}\right),
$$

where $\omega_{u}$ and $\omega_{d}$ are the two possible realisations of the binomial random variable $\omega$ given in (20), (so that $q=\frac{1}{2}$ ), and the pseudo-probability $q_{+}$is given by

$$
q_{+}=\frac{R(1+\lambda)-\omega_{d}}{\omega_{u}-\omega_{d}} .
$$

Inserting the expression for $\delta L^{*}$ into (A.1) gives the following representation for $H(T-\delta t, S, y)$ in the BUY region:

$$
H(T-\delta t, S, y)=-\exp (-\alpha y R S(1+\lambda))\left(\frac{q}{q_{+}}\right)^{q_{+}}\left(\frac{1-q}{1-q_{+}}\right)^{\left(1-q_{+}\right)}, \quad \text { in BUY }
$$


A similar analysis in the SELL region gives the optimal number of shares to sell, $\delta M^{*}$, as

$$
y-\delta M^{*}=y_{\mathrm{s}}(T-\delta t, S)=\frac{1}{\alpha S\left(\omega_{u}-\omega_{d}\right)} \log \left(\frac{q\left(1-q_{-}\right)}{(1-q) q_{-}}\right),
$$

where the pseudo-probability $q_{-}$is given by

$$
q_{-}=\frac{R(1-\mu)-\omega_{d}}{\omega_{u}-\omega_{d}}
$$

so that the value function in the SELL region is

$$
H(T-\delta t, S, y)=-\exp (-\alpha y R S(1-\mu))\left(\frac{q}{q_{-}}\right)^{q_{-}}\left(\frac{1-q}{1-q_{-}}\right)^{\left(1-q_{-}\right)}, \quad \text { in SELL. }
$$

Finally, in the NT region, the value function is given analytically by

$$
H(T-\delta t, S, y)=-\left[q \exp \left(-\alpha y S \omega_{u}\right)+(1-q) \exp \left(-\alpha y S \omega_{d}\right)\right], \quad \text { in NT. }
$$

\section{References}

Artzner, P., Delbaen, F., Eber, J.M., Heath, D., 1999. Coherent measures of risk. Mathematical Finance 9, $203-228$.

Avellaneda, M., Parás, A., 1994. Dynamic hedging portfolios for derivative securities in the presence of large transaction costs. Applied Mathematical Finance 1, 165-193.

Barrieu, P., El Karoui, N., 2002. Optimal design of derivatives in illiquid markets. Quantitative Finance 2, 181-188.

Bensaid, B., Lesne, J.P., Pagès, H., Scheinkman, J., 1992. Derivative asset pricing with transaction costs. Mathematical Finance 2, 63-86.

Black, F., Scholes, M., 1973. The pricing of options and corporate liabilities. Journal of Political Economy 81, 637-654.

Boyle, P.P., Tan, K.S., 1994. Lure of the linear. Risk 7 (4), 43-46.

Boyle, P.P., Vorst, T.C.F., 1992. Option replication in discrete time with transaction costs. Journal of Finance 47, 271-293.

Clewlow, L., Hodges, S.D., 1997. Optimal delta-hedging under transactions costs. Journal of Economic Dynamics and Control 21, 1353-1376.

Constantinides, G.M., 1986. Capital market equilibrium with transaction costs. Journal of Political Economy 94, 842-862.

Constantinides, G.M., Zariphopoulou, T., 1999. Bounds on prices of contingent claims in an intertemporal economy with proportional transaction costs and general preferences. Finance and Stochastics 3, 345-369.

Cox, J.C., Ross, S.A., Rubinstein, M., 1979. Option pricing: a simplified approach. Journal of Financial Economics 7, 229-263.

Cvitanić, J., Karatzas, I., 1999. On dynamic measures of risk. Finance and Stochastics 3, 451-482.

Cvitanić, J., Pham, H., Touzi, N., 1999. A closed-form solution to the problem of super-replication under transaction costs. Finance and Stochastics 3, 35-54.

Davis, M.H.A., 1997. Option pricing in incomplete markets. In: Dempster, M.A.H., Pliska, S.R. (Eds.), Mathematics of Derivative Securities. Cambridge University Press, Cambridge, UK.

Davis, M.H.A., Clark, J.M.C., 1994. A note on super-replicating strategies. Philosophical Transactions of the Royal Society of London A 347, 485-494.

Davis, M.H.A., Norman, A.R., 1990. Portfolio selection with transaction costs. Mathematics of Operations Research 15, 676-713. 
Davis, M.H.A., Zariphopoulou, T., 1995. American options and transaction fees. In: Davis, M.H.A., Duffie, D., Fleming, W.H., Shreve, S.E. (Eds.), Mathematical Finance-IMA Volumes in Mathematics and its Applications no. 65, Springer, New York.

Davis, M.H.A., Zervos, M., 1994. A problem of singular stochastic control with discretionary stopping. Annals of Applied Probability 4, 226-240.

Davis, M.H.A., Panas, V.G., Zariphopoulou, T., 1993. European option pricing with transaction costs. SIAM Journal of Control and Optimization 31, 470-493.

Dixit, A.K., 1991. A simplified treatment of the theory of optimal regulation of Brownian motion. Journal of Economic Dynamics and Control 15, 657-673.

Duffie, D., 1996. Dynamic Asset Pricing Theory, 2nd Edition. Princeton University Press, Princeton, NJ.

Dumas, B., 1991. Super contact and related optimality conditions. Journal of Economic Dynamics and Control $15,675-685$.

Dumas, B., 1992. Dynamic equilibrium and the real exchange rate in a spatially separated world. Review of Financial Studies 5, 153-180.

Dumas, B., Luciano, E., 1991. An exact solution to a dynamic portfolio choice problem under transaction costs. Journal of Finance 46, 577-595.

Edirisinghe, C., Naik, V., Uppal, R., 1993. Optimal replication of options with transactions costs and trading restrictions. Journal of Financial and Quantitative Analysis 28, 117-128.

Föllmer, H., Leukert, P., 2000. Efficient hedging: cost versus shortfall risk. Finance and Stochastics 4, $117-146$

Harrison, J.M., 1985. Brownian Motion and Stochastic Flow Systems. Wiley, New York, NY.

He, H., 1990. Convergence from discrete- to continuous-time contingent claims prices. Review of Financial Studies 3, 523-546.

Hodges, S.D., Neuberger, A., 1989. Optimal replication of contingent claims under transaction costs. Review of Futures Markets 8, 222-239.

Kabanov, Y.M., 1999. Hedging and liquidation under transaction costs in currency markets. Finance and Stochastics 3, 237-248.

Karatzas, I., Kou, S.G., 1996. On the pricing of contingent claims under constraints. Annals of Applied Probability 6, 321-369.

Kushner, H.J., 1990. Numerical methods for stochastic control problems in continuous time. SIAM Journal of Control and Optimization 28, 999-1048.

Kushner, H.J., 1997. Numerical methods for stochastic control problems in finance. In: Dempster, M.A.H., Pliska, S.R. (Eds.), Mathematics of Derivative Securities. Cambridge University Press, Cambridge, UK.

Kushner, H.J., Martins, L.F., 1991. Numerical methods for stochastic singular control problems. SIAM Journal of Control and Optimization 29, 1443-1475.

Lamberton, D., Pham, H., Schweizer, M., 1998. Local risk-minimization under transaction costs. Mathematics of Operations Research 23, 585-612.

Leland, H.E., 1985. Option pricing and replication with transactions costs. Journal of Finance 40, 1283-1301.

Levental, S., Skorohod, A.V., 1997. On the possibility of hedging options in the presence of transaction costs. Annals of Applied Probability 7, 410-443.

Mercurio, F., Vorst, T.C.F., 1997. Options pricing and hedging in discrete time with transaction costs. In: Dempster, M.A.H., Pliska, S.R. (Eds.), Mathematics of Derivative Securities. Cambridge University Press, Cambridge, UK.

Musiela, M., Zariphopoulou, T., 2001. Pricing and risk management of derivatives written on non-traded assets. Working paper, BNP Paribas, London.

Rouge, R., El Karoui, N., 2000. Pricing via utility maximization and entropy. Mathematical Finance 10, 259-276.

Shreve, S.E., Soner, H.M., 1994. Optimal investment and consumption with transaction costs. Annals of Applied Probability 4, 609-692.

Soner, H.M., Shreve, S.E., Cvitanić, J., 1995. There is no nontrivial hedging portfolio for option pricing with transaction costs. Annals of Applied Probability 5, 327-355.

Whalley, A.E., Wilmott, P., 1997. An asymptotic analysis of an optimal hedging model for option pricing with transaction costs. Mathematical Finance 7, 307-324. 